\title{
PRIMERAS JORNADAS IPERINAS: PRESENTACIÓN DE NUEVAS LÍNEAS DE INVESTIGACIÓN DEL INSTITUTO PIRENAICO DE ECOLOGÍA (CSIC)
}

\section{First Jornadas Iperinas: Presentation of new research topics of the Pyrenean Institute of Ecology}

I. Pardo*, J. Aranbarri, F. Barreiro, P. A. Bravo, H. A. Chaparro, F. Comín, R. Corriá, C. Español, M. Felipe-LuCía, M. Frugone, M. García-García, E. García-Prieto, G. Gil-Romera, I. Gimeno, L. Lasheras, A. Moreno, S. Palacio, A. Pérez-Sanz, S. Pironon, Y. Pueyo, F. Reig, H. Saiz, G. Sangüesa-Barreda, P. Tarrats,

\author{
J. ZABALZA \& J. REVUELTO*
}

Instituto Pirenaico de Ecología, CSIC, Campus de Aula Dei, Apartado 13034, 50059 Zaragoza, España * comparten primera autoría

RESUMEN.- En diciembre de 2012, se celebraron las primeras Jornadas IPErinas, reunión que permitió compartir al personal del Instituto Pirenaico de Ecología (CSIC), muchos de los trabajos de investigación que actualmente se están llevando a cabo por los distintos grupos del centro. Estas Jornadas surgieron de la necesidad de compartir y difundir los principales objetivos y resultados más relevantes de nuevas líneas de investigación desarrolladas actualmente en el centro, como parte fundamental de la comunicación científica y transferencia de conocimiento a la sociedad. Bajo estas líneas, se presenta una reseña de la las primeras Jornadas IPErinas, a través de un breve resumen de las charlas presentadas.

Palabras clave: Instituto Pirenaico de Ecología, CSIC, nuevas líneas de investigación, divulgación científica, transferencia conocimiento, colaboración científica. 
ABSTRACT.- In December 2012, the first Jornadas IPErinas took place at the Pyrenean Institute of Ecology (CSIC). The aim of this meeting was to show many of the new research topics that nowadays are being developed in the Institute. This workshop grew out of the need to disseminate the main new research lines developed at the center, as a fundamental part of scientific communication and knowledge transfer to society. In this paper, we briefly review the topics presented in the first meeting Jornadas IPErinas, summarizing the oral contributions.

Keywords: Pyrenean Institute of Ecology, CSIC, new research lines, science dissemination, knowledge transfer, scientific collaboration.

\section{Introducción}

El Instituto Pirenaico de Ecología (IPE), centro integrado en el Consejo Superior de Investigaciones Científicas (CSIC), tiene dos sedes en la Comunidad Autónoma de Aragón, situadas una en Jaca y otra en el Campus de Aula Dei de Zaragoza. El IPE es el Instituto del CSIC más antiguo de Aragón y el único dedicado exclusivamente a la investigación medio ambiental y territorial.

El IPE tal como se conoce hoy en la actualidad, tomó forma en el año 1990, sin embargo sus orígenes se remontan a 1942. Tanto el nombre del centro, como los departamentos que lo componen y las líneas de investigación, han tenido una evolución constante durante los más de 70 años de historia del Instituto (Martínez-Rica, 2005). A lo largo de este tiempo, el IPE ha desarrollado un elevado volumen de trabajos de investigación sobre la cordillera pirenaica, si bien durante las últimas décadas las áreas de trabajo fueron ampliándose a muy diversas zonas a nivel mundial, en las que actualmente se desarrollan proyectos y / o colaboraciones científicas (Figura 1).

La misión principal del IPE es contribuir a la comprensión científica del planeta Tierra y los seres que lo habitan, estudiando la estructura y funcionamiento de los sistemas naturales y su modificación como respuesta a la actividad humana, de manera especial en las áreas de montaña, y su relación con el Cambio Global. El progreso en este conocimiento se asienta en la comprensión de la organización y funcionamiento de los sistemas naturales terrestres, incluyendo los aspectos biológicos, hidrológicos, climáticos, geomorfológicos y antropogénicos a escalas temporales que comprenden, desde los ciclos glaciares/interglaciares durante el Cuaternario, hasta la monitorización anual de nuestros ecosistemas y especies (http:/ / www.ipe.csic.es/).

Para alcanzar este objetivo, la estructura actual del IPE (véase http://www.ipe.csic.es/organigrama) consta de varias líneas de 


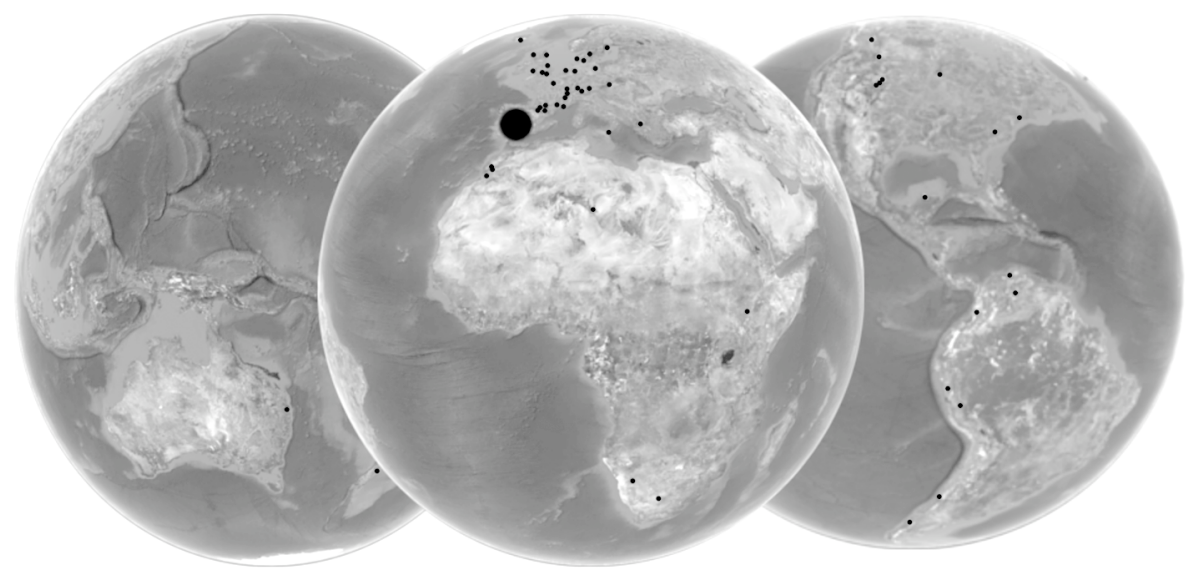

Figura 1. Localidades (puntos negros) de trabajo en las que los miembros del Instituto Pirenaico de Ecología (CSIC) desarrollan sus investigaciones.

Figure 1. Locations (red points) where the Pyrenean Institute of Ecology staff carries out its work.

investigación, aglutinadas en dos grandes departamentos: “Conservación de la Biodiversidad y Restauración de Ecosistemas" (http:/ / www. ipe.csic.es/ conservacion-biodiversidad) y "Procesos Geoambientales y Cambio Global" (http://www.ipe.csic.es/procesos-geoambientales), que difieren en metodología y espacio temporal de trabajo, pero persiguen un objetivo de conocimiento común. De esta manera, la problemática del Cambio Global se aborda desde un punto de vista holístico, dando cobijo a un nutrido y diverso grupo de científicos con formaciones muy diversas y complementarias (geógrafos, químicos, biólogos, geólogos, físicos, ingenieros, ambientólogos, etc.). Con la finalidad de fomentar la colaboración interdepartamental y progresar en un avance científico común se celebran, con distinta frecuencia temporal, seminarios, reuniones interdepartamentales y "journal clubs" (sesiones para comentar artículos científicos en grupo), así como claustros científicos. Precisamente, es en este contexto de colaboración donde se enmarcan las primeras Jornadas IPErinas.

\section{Las jornadas IPErinas 2012}

Como complemento a las actividades anteriormente mencionadas y que ya están implementadas en el Instituto, en diciembre de 2012 se organizó la primera edición de las Jornadas IPErinas a iniciativa de un grupo del personal 
investigador en formación. El principal propósito no fue otro que el de dar a conocer mejor cuáles son las investigaciones que se están desarrollando actualmente en el Instituto. Con esta iniciativa, se ha pretendido favorecer el intercambio de aproximaciones científicas para, en definitiva, fomentar la trasferencia de información a todos los niveles del Instituto. Las jornadas se plantearon como una sucesión de breves charlas (10 minutos cada una), con un carácter informal y en un ambiente distendido pero sin perder el rigor científico. Todo el personal del centro estuvo invitado a tomar parte en ellas y exponer su función en el centro, esto es, el qué, dónde, cómo, por qué y objetivos de su labor. El resultado final fueron más de 25 charlas impartidas por un nutrido grupo de investigadores, técnicos y personal investigador en formación (quienes representaron el colectivo más numeroso).

A continuación se expone un breve resumen de las jornadas, que dan buena muestra de hacía dónde están dirigidas algunas de las nuevas líneas de investigación del IPE, así como de la diversidad de trabajos que se realizan en el Instituto (Figura 2). No obstante, no todas las líneas de investigación fueron abordadas durante las jornadas con igual profundidad (en particular las más tradicionales y consolidadas), por lo que el resumen debe entenderse como un reflejo parcial de todo el trabajo que se desarrolla en el centro.

\subsection{La variabilidad climática terrestre; la gran reguladora de los ecosistemas y los procesos geomorfológicos}

El Cambio Global incluye la variabilidad climática natural y la inducida por las actividades humanas, así como los cambios provocados por ambos impactos en los ecosistemas terrestres y en los ciclos bio-geoquímicos. La actividad del departamento de "Procesos Geoambientales y Cambio Global" se enmarca precisamente en comprender los procesos de Cambio Global y sus efectos en la dinámica hidrológica y geomorfológica del territorio, a distintas escalas espacio-temporales. Dos grandes grupos de investigación constituyen este departamento, "Hidrología Ambiental" y "Paleoambientes Cuaternarios", con un propósito común, el estudio del Cambio Global desde perspectivas temporales diferentes, pero complementarias: pasado, presente y futuro.

Los estudios presentados por los integrantes del grupo de "Hidrología Ambiental", están encaminados a analizar la variabilidad climática y las relaciones entre Cambio Global y funcionamiento hidrológico y geomorfológico del territorio, a diferentes escalas espaciales, desde laderas a grandes cuencas. La variabilidad de la circulación atmosférica y los procesos de calentamiento global afectan directamente al volumen de precipitación, los 


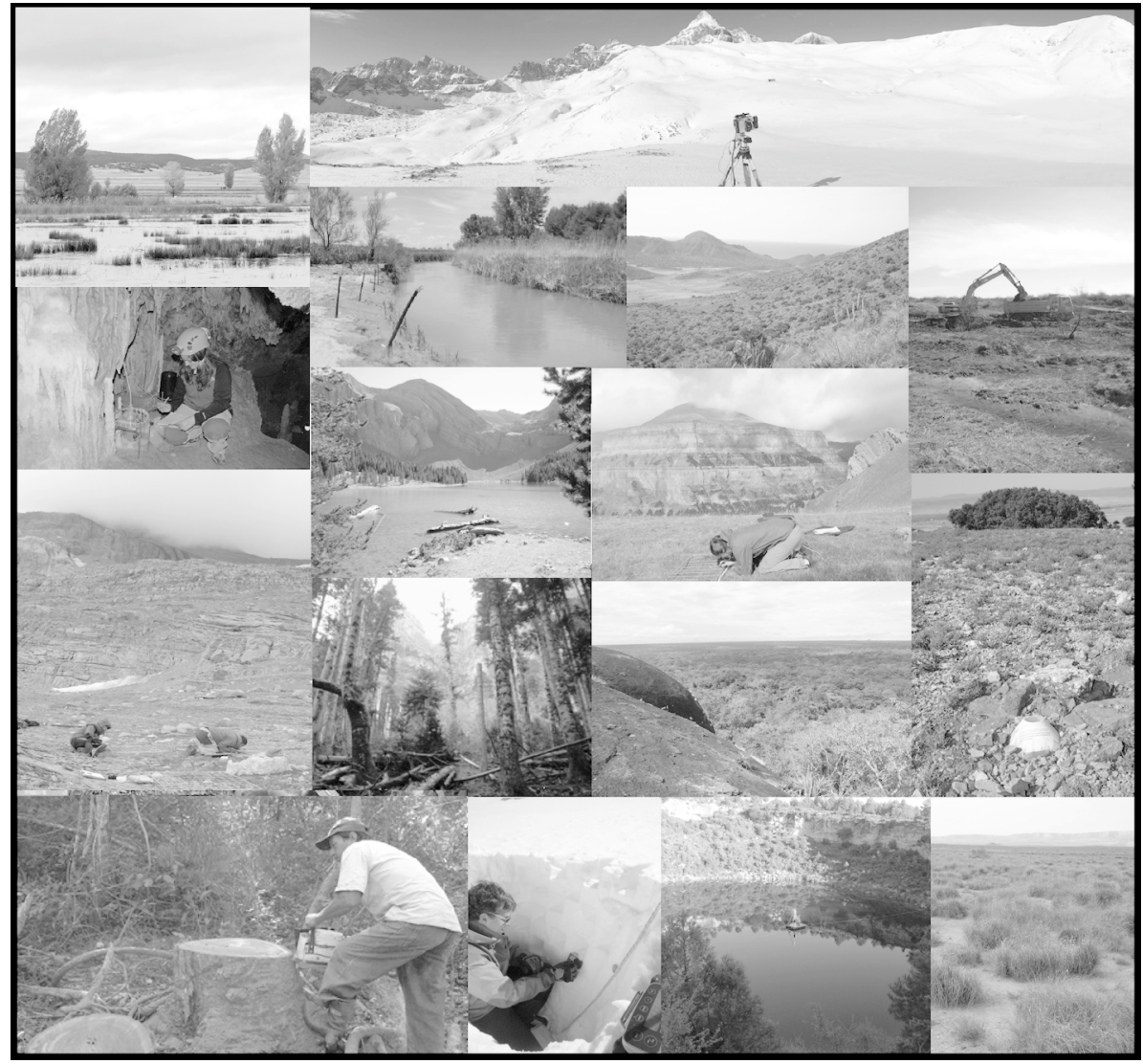

Figura 2. Imágenes de los diferentes ambientes objeto de estudio por parte del personal del Instituto Pirenaico de Ecología (CSIC).

Figure 2. Images of different working environments studied by the Pyrenean Institute of Ecology. 
procesos de acumulación y fusión de nieve, el régimen fluvial, la magnitud y frecuencia de avenidas y sequías, y la intensidad de la evapotranspiración. Así mismo, otros procesos como la partición de la precipitación, la evolución de las reservas de agua en el suelo, el coeficiente de escorrentía y, finalmente, el caudal de los ríos, se ven influidos indirectamente por la citada variabilidad. Todo esto condiciona sobremanera la gestión y el aprovechamiento de los recursos hídricos.

En este contexto, algunos integrantes del grupo dirigen sus esfuerzos a la modelización climática, usando modelos hidrológicos espacialmente distribuidos en los que se relacionan los patrones climáticos e hidrológicos con patrones espaciales de vegetación. Estos modelos resultan muy interesantes para el conocimiento de la sensibilidad de los ecosistemas ante futuros cambios climáticos y de usos de suelo. El modelo RHESSys (Regional Hydro-Ecologic Simulation System), está especialmente diseñado para simular de manera integrada este tipo de procesos a escala de cuenca. Su implementación en cuencas del Pirineo, ayuda a predecir futuros comportamientos hidrológicos y de la vegetación ante los distintos escenarios de cambio climático (LópezMoreno et al., enviado). Otra labor llevada a cabo por integrantes de este grupo, es el desarrollo de una web para la consulta del SPEI, (Índice estandarizado de precipitación y evapotranspiración, disponible a escala mundial desde los años 50 hasta la actualidad: Vicente-Serrano et al., 2010) y el cálculo del NDVI (Índice normalizado de diferenciación de la vegetación, para la Península Ibérica: Alatorre et al., 2010) en el mismo periodo.

El estudio de la nieve desde un punto de vista hidrológico, centra también parte de los esfuerzos de estos investigadores. El propósito es determinar los procesos que rigen la acumulación y fusión del manto de nieve, así como estimar la cantidad de agua acumulada en estado sólido en las cuencas experimentales que se están monitorizando mediante tecnología Láser Escáner Terrestre (Revuelto et al., 2013). De este modo, a medida que se profundiza en el conocimiento de esta variable, se posibilita la mejora de la capacidad predictiva de los citados modelos (López-Moreno et al., 2012).

Para una mejor comprensión de las proyecciones futuras de los modelos climáticos, resulta imprescindible mejorar el conocimiento de las distintas etapas climáticas que han afectado al planeta en el pasado, área de investigación en la que está especializado el grupo de "Paleoambientes Cuaternarios". Este equipo de investigadores está centrado en el estudio de la paleoclimatología y la paleoecología, disciplinas que intentan caracterizar los cambios ambientales pasados y entender las respuestas de los ecosistemas a estos cambios, en escalas temporales superiores a décadas o siglos. Con ello se persigue la reconstrucción de la dinámica del clima, la hidrología y las comunidades vegetales esencialmente, centrando la atención, en los cambios 
abruptos (Valero-Garcés et al., 2000; González-Sampériz et al., 2010; Moreno et al., 2010), y en la resiliencia de los sistemas ecológicos (Gil-Romera et al., 2010), tanto en ausencia como en presencia del ser humano. De este modo, los resultados obtenidos son utilizados para evaluar la capacidad de respuesta de los ecosistemas actuales pueden tener ante al Cambio Global.

Los estudios presentados en las charlas, permitieron comprender la diversidad de trabajos que aglutina este grupo, de filosofía "multiproxy" ó multi-paramétrica. La Basa de la Mora, ibón situado en el macizo pirenaico de Cotiella, es un claro ejemplo de los objetivos y metodología llevados a cabo por estos investigadores. El estudio multi-paramétrico desarrollado en este registro sedimentario, se centra esencialmente en el estudio del polen fósil, la geoquímica del sedimento y los restos de quironómidos, ha permitido reconocer los rápidos y profundos cambios climáticos acaecidos en esta zona del Pirineo durante los últimos 10.000 años, poniendo de manifiesto la extraordinaria sensibilidad de nuestras montañas a los cambios ambientales (Pérez-Sanz et al., 2013). Además, la identificación de partículas de carbón fósil a lo largo de una secuencia sedimentaria, está permitiendo estudiar la relación existente entre el fuego, los ecosistemas y el clima en escalas temporales largas, así como conocer la respuesta de la vegetación a esta perturbación e identificar los umbrales que provocan cambios irreversibles en la composición vegetal (Lasheras-Álvarez et al., 2013). Otros ibones pirenaicos estudiados por este grupo en la actualidad son el de Marboré y Urdiceto, que junto con la Basa de la Mora, están permitiendo reconstruir la evolución limnológica de tres lagos de altitud durante los últimos 2000 años con muy alta resolución. En esta línea, se está estudiando también la comunidad actual de macroinvertebrados bentónicos, con especial atención a la familia de los quironómidos, paleoindicador biológico utilizado básicamente para reconstruir cambios de temperatura y del nivel del agua de las cuencas lacustres.

Otras lagunas, con características distintas a los ibones pirenaicos, tienen también interés para recabar información paleoambiental. Tal es el caso de la laguna del Cañizar de Villarquemado, en Teruel, cuya secuencia sedimentaria constituye un registro excepcional que cubre más de 130.000 años de historia. La variabilidad climática en esta localización se ha visto condicionada por la continentalidad a escala milenaria, potenciando la resiliencia de la dinámica vegetal, y una particular evolución paleohidrológica, muy determinada a su vez por la geomorfología de la cuenca y su localización intramontañosa (Aranbarri et al., 2013). Por otra parte, en esta misma zona se está monitorizando en la actualidad la lluvia polínica para tratar de establecer la relación existente entre el polen depositado en una cuenca sedimentaria y la vegetación real de su entorno. A partir de estos datos se pueden proponer 
reconstrucciones paleoambientales más precisas, además de permitirse inferencias espaciales del paisaje y planteamientos de escenarios de cambio climático y dinámica de la vegetación a lo largo del tiempo (García-Prieto et al., 2012).

Continuando en la Península Ibérica, los lagos kársticos formados en dolinas (llamados localmente torcas) del Tejo, la Parra y Laguna del Tobar (Cuenca) también están siendo objeto de estudio. En ellos se estudia la respuesta hidrológica y geoquímica al impacto humano y al cambio climático durante los últimos 4000 años (Barreiro-Lostre et al., 2011). Por medio de sondeos de sedimento, se pretende comprender mejor los procesos de erosión, transporte y sedimentación en los sistemas lacustres de origen kárstico. Además, a partir de estudios sedimentológicos, geoquímicos y biológicos, es posible conocer la respuesta de los sistemas lacustres a los cambios globales del pasado (climáticos y antropogénicos), bajo escenarios de condiciones semejantes a las presentes.

Pero no sólo se trabaja en territorio peninsular, integrantes de este equipo centran su labor en Sudamérica. En los lagos del Chile central semiárido de la actualidad, una de zona particularmente frágil frente al Cambio Global, se estudian las relaciones ambientales a distintas escalas temporales y espaciales, con el propósito de comprender la historia climática más allá de los registros instrumentales.

Dejando a un lado los sistemas acuáticos, las cuevas son una fuente muy interesante de información del clima pasado. A través del análisis composicional de espeleotemas (estalagmitas y estalactitas), es posible reconstruir la evolución del clima. Las estalagmitas son registros excepcionales del Cambio Global porque albergan información, tanto de la temperatura como de la disponibilidad hídrica que puede extraerse a partir del análisis de sus isótopos y su contenido en elementos traza (Moreno et al., 2010). Debido a la complejidad de los sistemas kársticos, buena parte de la investigación se centra en el seguimiento de variables ambientales (dentro y fuera de la cueva). El elevado número de cuevas estudiadas con esta metodología se distribuyen por muy diferentes localidades (Asturias, Málaga, Menorca, Soria y Burgos), si bien la mayoría de ellas se localizan principalmente en el Pirineo y la Cordillera Ibérica.

\subsection{Las consecuencias del Cambio Global en montañas, bosques, estepas y ecosistemas acuáticos}

Si bien en las charlas de la sección precedente se presentaron evidencias de la magnitud que han tenido y están teniendo los cambios climáticos y 
antropogénicos, los estudios que se presentan a continuación abordan las consecuencias que el Cambio Global está teniendo sobre la estructuración actual y funcionamiento de la biodiversidad y de los ecosistemas. Los estudios que se vienen realizando en el departamento de "Conservación de la Biodiversidad y Restauración de Ecosistemas", están focalizados a ambientes excepcionalmente interesantes por su singularidad en términos de biodiversidad y/o grado de amenaza, tales como la alta montaña, los ecosistemas semi-áridos, los ríos y humedales de la Península Ibérica. Además, recientemente se ha iniciado una línea de trabajo en bosques tropicales secos de América del Sur.

Como punto de partida para evaluar las consecuencias de los cambios ambientales, es necesario conocer la estructura de los diferentes aspectos de la biodiversidad, así como la capacidad de las comunidades y especies para ajustar su funcionamiento a los cambios ambientales (clima, edafología, cambio de uso del suelo u otras perturbaciones) a los que están sometidos. Tal y como se puso de manifiesto durante las Jornadas IPErinas, en este Instituto se utilizan aproximaciones muy diversas, que van desde la autoecología (fenología, crecimiento primario y secundario, ciclado de nutrientes, agua y carbono) y morfología de las especies, hasta la ecología de poblaciones y comunidades.

En los pastos alpinos de alta montaña, la huella de cambios ambientales recientes se está determinando a partir de la combinación de datos históricos y monitorizaciones permanentes de comunidades de plantas. Si bien hay alguna pequeña muestra de cambio, la intensidad con que éstos ocurren es sorprendentemente lenta, contrastando con las predicciones más catastrofistas (García et al., 2012). La recolección de información detallada en el campo (p. ej. co-ocurrencia de especies a micro escala, tasas vitales, etc.), es sin duda una gran herramienta para generar modelos de distribución más realistas. En este aspecto, se está trabajando para intentar conocer la evolución de los límites de distribución de las especies en el contexto del actual de Cambio Global (Pironon, enviado).

En el ecotono pasto-bosque subalpino, los cambios en la diversidad vegetal también ocurren lentamente, incluso cuando la cubierta forestal aumenta en su límite superior de distribución altitudinal (Pardo et al. en revisión). Además, como paso previo a la reforestación del piso subalpino, se observan procesos de matorralización en terrenos que fueron adecuados para el pastoreo, hoy venido a menos (Komac, 2010). Sin embargo, a pesar de que ciertos factores como el clima o el cambio de uso del suelo están promoviendo la expansión o recuperación de las masas forestales en la montaña, es una constatación que estos mismos factores están causando una mortalidad acusada en varias especies arbóreas, tanto en este tipo de bosques montanos 
como en otros de todo el mundo. En particular, esta mayor mortandad se ha asociado a diversos estresores como factores climáticos (sequía), o agentes bióticos (insectos defoliadores como la procesionaria del pino, insectos perforadores como los escolítidos, plantas hemiparásitas como el muérdago, hongos patógenos). Por lo tanto, resulta necesario conocer cuál es la contribución real de estos agentes, así como la respuesta de los árboles afectados. Estas cuestiones se están intentando resolver por medio de métodos de dendroecología (Sangüesa-Barreda et al., 2012), y de aproximaciones más fisiológicas, como el estudio del uso del carbono (Palacio et al., 2012a).

En otro tipo de formaciones forestales, como es el caso del bosque tropical seco de Bolivia, el principal determinante de la dinámica del bosque es la propia gestión maderera. En estos lugares se está determinando la estructura y dinámica del crecimiento secundario de varias especies arbóreas a distintas escalas temporales, evaluando su relación con variables demográficas y climáticas mediante la datación de discos (Mendivelso et al., 2012). Por medio de estos estudios, se están asentando las bases científicas para mejorar la gestión forestal que se está realizando actualmente. Asimismo, ecosistemas de riqueza tan notable como el bosque tropical seco son un laboratorio ideal para tratar de descifrar qué mecanismos bióticos y abióticos facilitan la coexistencia de especies arbóreas. Para ello, se utilizan mapas de poblaciones de árboles a distintas escalas espaciales, desde censos forestales de 1000-4000 ha, hasta parcelas permanentes de 1 ha, y se aplican técnicas de análisis espacial (Corrià et al., 2012).

Dentro del grupo de "Conservación de Ecosistemas Naturales", existe también una línea centrada en el estudio del funcionamiento y la estructura de los ecosistemas semi-áridos. En estos ambientes de condiciones muy limitantes, tanto las interacciones entre especies como los mecanismos de adaptación al medio, son fundamentales para la supervivencia de las mismas. Por ello, los investigadores de este grupo están analizando, entre otras cuestiones, la relación entre el funcionamiento hidrológico del ecosistema y el patrón espacial de la vegetación, y cómo este último puede utilizarse como indicador del estado de conservación de los ecosistemas semi-áridos (Pueyo et al., 2012). Las interacciones bióticas están siendo abordadas mediante una aproximación novedosa para descifrar la estructura y dinámica de estos ecosistemas, basada en la aplicación de la teoría de redes de interacción (Saiz \& Alados, 2012). Finalmente, dentro de los ecosistemas semi-áridos, los sistemas de yesos constituyen uno de los ambientes más restrictivos para la vida de las plantas. Mediante una aproximación ecofisiológica y funcional, basada en el estudio de los procesos ecológicos a nivel de la planta completa, se están analizando los mecanismos que presentan las plantas exclusivas y no exclusivas de los yesos para sobrevivir en las duras condiciones imperantes en estos sistemas (Palacio et al., 2007; 2012b). 
Respecto a los ecosistemas acuáticos, el grupo de "Restauración Ecológica" lleva a cabo diferentes estudios para evaluar la efectividad de la restauración o construcción de humedales para actuar como fuente de especies y funciones en llanuras de inundación degradas. Para ello, se están analizando las características estructurales (p.ej. diversidad de especies, calidad fisicoquímica del agua) y funcionales (metabolismo, rasgos biológicos funcionales, intercambio de gases, etc.) en una serie de humedales naturales remanentes y humedales construidos / restaurados, de la llanura de inundación del tramo medio del río Ebro. Asimismo, se está evaluando la influencia de diferentes factores ambientales en la respuesta taxonómica y funcional de los humedales para establecer, en última instancia, las bases científico-técnicas de los proyectos de restauración ecológica con el objetivo de conseguir humedales multifuncionales y dar un mejor uso a los recursos invertidos en dichos proyectos (Español et al., 2012).

Siguiendo en el medio acuático, en las Jornadas se presentó una problemática que resulta de la interacción biótica y climática, y que está tomando gran relevancia en las últimas décadas: la introducción y expansión del mejillón cebra (Navarro et al., 2006). En particular, esta línea de trabajo persigue identificar las principales variables de influencia que estarían determinando la distribución espacial y temporal de esta especie en la cuenca del Ebro, mediante el ajuste de modelos aditivos generalizados que permitan explicar esta relación y llegar a obtener predicciones.

\subsection{Mitigando la degradación de los ecosistemas: criterios cientificos de gestión}

En muchas ocasiones, el conocimiento científico (o el mal uso que se hace de él), no es suficiente para evitar la degradación y/o transformación de los ecosistemas y la pérdida de biodiversidad. En esta situación, la restauración de los ecosistemas es, si no la única, la mejor de las alternativas, no sólo para mitigar la degradación biológica, paisajística y cultural, sino para promover el beneficio que la sociedad puede obtener de dicho ecosistema -servicios ecosistémicos- (Trabucchi et al., 2012). Para este objetivo, es necesario fusionar el conocimiento científico y los aspectos sociales y económicos, aspectos con los que trabajan en el grupo de "Restauración Ecológica". Dicha tarea, se viene realizando a través de una novedosa aproximación multiescalar (Trabucchi et al., 2013), que está permitiendo proponer aplicaciones prácticas para la gestión de los usos del suelo de las llanuras de inundación (o agroecosistemas de ribera), con el fin de optimizar la provisión de servicios del ecosistema al conjunto de la sociedad (Felipe-Lucia \& Comín, 2012). 


\section{Conclusiones}

La valoración unánime del desarrollo de las primeras Jornada IPErinas fue muy positiva. El principal objetivo (mejorar el conocimiento de las los trabajos de investigación que se están desarrollando actualmente en el centro) se logró con éxito gracias a la elevada participación del personal. Además, tal y como quedó reflejado en la evaluación conjunta, las Jornadas sirvieron para dar muestra del gran potencial existente en el centro para desarrollar colaboraciones y trabajos conjuntos.

Sin duda, y tal y como se hizo explícito en la valoración, el formato elegido para la celebración de las Jornadas IPErinas fue acertado. No obstante, sería muy positivo plantear futuras ediciones en un lugar abierto para otro tipo de público, como por ejemplo la Universidad, donde este tipo de divulgación de la labor científica tiene cabida.

En conclusión, este tipo de actividad resulta enriquecedora para todos participantes, sin embargo, debe entenderse como una más de un conjunto de actividades destinadas a la comunicación de la ciencia a diferentes niveles. Es decir, es una pieza más del puzle de la divulgación científica, donde los principales beneficiarios son los propios participantes. Por esta razón, animamos a cualquier centro de investigación a realizar este tipo de actividades, ya que el esfuerzo adicional que exige su preparación es muy inferior al beneficio reportado (tanto desde un punto de vista científico, como personal). La divulgación de los resultados científicos constituye una de las primeras maneras de retornar los recursos destinados a la ciencia a la sociedad y, sin embargo, es una tarea que plantea dificultades: saber explicar una investigación de manera sencilla y con un lenguaje de fácil compresión, pero eficaz, es esencial. Creemos que actividades como ésta, donde un científico de una determinada área del conocimiento, transmite su labor y metodología a otros de un perfil diferente, es un primer paso para iniciarse en la tarea de la divulgación.

\section{Agradecimientos}

A todo el personal de IPE que de una $u$ otra manera (impulsando, participando, asistiendo) contribuyó a dar forma a la primera edición de las Jornadas IPErinas. En particular, nos gustaría agradecer a Penélope GonzálezSampériz y Adela Lamana su apoyo en la realización de este manuscrito. Asimismo hacemos extensivo nuestro agradecimiento a todo el personal del IPE (administrativos, técnicos de campo y laboratorio, investigadores, estudiantes de máster, etc.), que por motivos de diversa índole no asistieron 
a las Jornadas IPErinas 2012, pero que contribuyen en el día a día a realizar una ciencia de calidad en nuestro centro.

\section{Referencias}

Alatorre, LC., Beguería S. \& Vicente-Serrano, SM., 2010. Análisis de la evolución espacio-temporal del NDVI sobre áreas vegetadas y zonas de riesgo de erosión en el Pirineo Central. Pirineos, 165: 7-27.

Aranbarri, J., González-Sampériz, P., Valero-Garcés, B., Moreno, A., GilRomera, G., Sevilla-Callejo, M., Di Rita, F., García-Prieto, E., Magri, D., Carrión, JS. \& Morellón, M., en prensa. Postglacial vegetation dynamics and climate variability inferred from Villarquemado paleolake sequence (Iberian Range, north-eastern Spain).Global and Planetary Change.

Barreiro-Lostres, F., Moreno, A. \& Valero-Garcés, B., 2011. Facies sedimentarias de la laguna kárstica de La Parra (Cuenca) durante los últimos 1600 años cal. BP. Geogaceta, 50: 109-112.

Corrià, R., Pélissier, R., Camarero, JJ. \& Toledo, M., 2012. Spatial patterns of trees are related with species traits in a Bolivian tropical dry forest. 49th Annual Meeting of the Association for Tropical Biology and Conservation (ATBC) Ecology, Evolution and Sustainable Use of Tropical Biodiversity. 1922/06/2012, Bonito, Brazil.

Español C., Gallardo B., Martín A., Pino M.R. \& Comín FA., 2012. Is net ecosystem production higher in natural relative to constructed wetlands? Aquatic Sciences, DOI: 10.1007/s00027-012-0284-1.

Felipe-Lucia, MR. \& Comín, FA., 2012. Ecosystem Services Evaluation in Riparian Habitats: the case of the Piedra River (NE Spain). Poster presentation. 4th International EcoSummit, Columbus, Ohio, USA.

García, MB., Pardo, I., Pata, MP., Camarero, JJ., Gómez, D., García-González, R., Errea, P., Pironon, S., Aldezábal, A., Olesen, JM., Roquet, C. \& Lavergne, S. 2012. Monitorización de la biodiversidad vegetal y sus distintos componentes, en un Parque Nacional de montaña. Proyectos de Investigación en Parques Nacionales 2008-2011.

García-Prieto, E., 2012. Pollen Productivity Estimates in Mediterranean landscapes: modelling the spatial distribution of past vegetation at $\mathrm{El}$ Cañizar de Villarquemado palaeolake (Teruel, NE SPAIN). Quaternary International, 279-280:160.

Gil-Romera, G., Lamb, HF., Turton, D., Sevilla-Callejo, M. \& Ummer, M., 2010. Long-term resilience, bush encroachment patterns and local knowledge in a Northeast African savanna. Global Environmental Change, 20: 612-626

González-Sampériz, P., Leroy, S., Carrión, J; Fernández, S., García-Antón, M., Gil-García, M., Uzquiano, P., Valero-Garcés, B., \& Figueiral, I., 2010. 
Steppes, Savannahs, Forests and Phytodiversity Reservoirs during the Pleistocene in the Iberian Peninsula. Review of Palaeobotany and Palynology, 162 (3): 427-457. DOI: 10.1016/j.revpalbo.2010.03.009

Komac, B., 2010. Effets des modifications de l'utilisation des terres sur la conservation des pâturages subalpins du Parc National de Ordesa Mont-Perdu. 2010. PhD.

Lasheras-Álvarez, L., Pérez Sanz, A., Gil-Romera, G., González-Samperiz, P., Sevilla-Callejo, M. \& Valero-Garcés, B. en prensa. Historia del fuego y la vegetación en una secuencia holocena del Pirineo Central: La Basa de la Mora. Cuadernos de Investigación Geográfica.

López-Moreno, JI., Pomeroy, J., Revuelto, J. \& Vicente-Serrano, SM., 2012. Response of snow processes to climate change: Spatial variability in a small basin in the Pyrenees. Hydrological Processes. DOI: 10.1002/ hyp.9408.

Lopez-Moreno, JI., Vicente-Serrano, SM., Zabalza, J., Revuelto, J., Gilaberte, M., Azorín, C., Morán-Tejeda, E., García-Ruiz, JM., Tague, C., enviado. Climate and land use change impacts in water availability and reservoir management in the upper Aragon river: a Mediterranean mountain basin. Science of the Total Environment.

Martinez-Rica, J. P., 2005. Discurso del director del Instituto Pirenaico de Ecología. Pirineos, 160: 165-176.

Mendivelso HA., Camarero JJ., Royo O., Gutierrez E. \& Toledo M., enviado. Divergent growth responses to water availability of coexisting tree species are linked to contrasting wood water storage in a Bolivian tropical dry forest. Journal of Ecology.

Moreno, A., Stoll, H. M., Jiménez-Sánchez, M., Cacho, I., Valero-Garcés, B., Ito, E., y Edwards, L. R., 2010. A speleothem record of rapid climatic shifts during last glacial period from Northern Iberian Peninsula. Global and Planetary Change 71: 218-231.

Navarro, E., Bacardit, M., Caputo, L., Palau, T. \& Armengol, J., 2006. Limnological characterization and flow patterns of a three-coupled reservoir system and their influence on Dreissena polymorpha populations and settlement during the stratification period. Lake and Reservoir Management 22 (4): 293-302.

Palacio S., Escudero A., Milla R., Maestro M., Montserrat-Martí G. \& Albert M.J., 2007. Plants living on gypsum: beyond the specialist model. Annals of Botany, 99: 333-343.

Palacio, S., Hernández, R., Maestro-Martínez, M. \& Camarero, J.J., 2012a. Fast replenishment of initial carbon stores after defoliation by the pine processionary moth and its relationship to the re-growth ability of trees. Trees - Structure and Function: 1-14.

Palacio S., Johnson D., Escudero A. \& Montserrat-Martí G., 2012b. Root 
colonization by AM fungi differs between gypsum specialist and nonspecialist plants: links to the gypsophile behaviour. Journal of Arid Environments, 76: 128-132.

Pardo I., Camarero J.J., Gutiérrez E. \& García, M.B., en revision. Minor and spatially uncoupled changes in tree cover and ground vegetation at two Pyrenean treelines over 11 years. Plant Ecology and Diversity

Pironon, S., Villellas, J., Morris, WF., Doak, DF. \& Garcia, M.B., enviado: The 'center-periphery hypothesis': a biogeographical paradigm revisited. Ecology letters.

Pérez-Sanz, A., González-Sampériz, P., Moreno, A., Valero-Garcés, B., GilRomera, G., Rieradevall, M., Tarrats, P., Lasheras-Álvarez, L., Morellón, M., Belmonte, A., Sancho, C., Sevilla-Callejo, M. \& Navas, A., en prensa. Climate variability, vegetation dynamics and fire regimen in the Central Pyrenees: the Basa de la Mora sequence (NE Spain). Quaternary Science Reviews.

Pueyo, Y., Moret-Fernández, D., Sáiz, H., Bueno, CG. \& Alados, CL., 2012. Relationships between plant spatial patterns, water infiltration capacity and plant community composition in semi-arid Mediterranean ecosystems along stress gradients. Ecosystems (DOI: 10.1007/s10021-0129620-5)

Revuelto, J., López-Moreno, JI., Azorín-Molina, C., Arguedas, G., VicenteSerrano, S.M. \& Serreta, A., en prensa. Utilización de técnicas láser escáner terrestre en la monitorización de procesos geomorfológicos dinámicos: el manto de nieve y heleros en áreas de montaña. Cuadernos de Investigación Geográfica.

Saiz, H. \& Alados, CL., 2012. Changes in Semi-Arid Plant Species Associations along a Livestock Grazing Gradient. PLoS ONE 7(7): e40551. doi:10.1371/journal.pone.0040551.

Sangüesa-Barreda, G., Linares, JC., \& Camarero, JJ., 2012. Mistletoe effects on Scots pine decline following drought events: insights from within-tree spatial patterns, growth and carbohydrates. Tree Physiology 32: 585-598. doi:10.1093/treephys/tps031.

Trabucchi, M., Ntshotsho, P., O’Farrell, PJ. \& Comín, FA., 2012. Ecosystem service trends in basin-scale restoration initiatives: A review. Journal of Environmental Management, 111: 18-23.

Trabucchi, M., Comín, FA. \& O'Farrell, PJ., 2013. Hierarchical priority-setting for restoration in a watershed in NE Spain, based on assessments of soil erosion and ecosystem services. Regional Environmental Change, DOI:10.1007/s10113-012-0392-4

Valero-Garcés, BL., González-Sampériz, P., Delgado-Huertas, A., Navas, A., Machín, J. \& Kelts, K., 2000. Late Glacial and Late Holocene environmental 
I. PARDO, J. ARANBARRI, F. BARREIRO, P. A. BRAVO, H. A. CHAPARRO, F. COMÍN, R. CORRIÁ...

and vegetational change in Salada Mediana, Central Ebro Basin, Spain. Quaternary International A 73/74, 29-46.

Vicente-Serrano, SM., Beguería, S., López-Moreno, JI., Angulo, M. \& El Kenawy, A., 2010. A new global $0.5^{\circ}$ gridded dataset (1901-2006) of a multiscalar drought index: comparison with current drought index datasets based on the Palmer Drought Severity Index. Journal of Hydrometeorology 11 (4): 1033-1043.

Villar, L. \& Valles, L., 1995. Cincuenta años de estudios Pirenaicos. Historia Natural 93, Ed. Instituto Pirenaico de Ecología- Instituto de Estudios Altoaragoneses, 255-274 pp. 